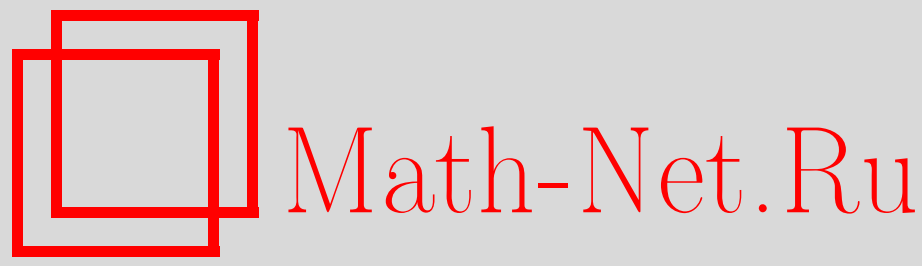

Т. В. Рябуха, О функционалах для средних значений наблюдаемых одномерных бесконечных систем частиц, TMФ, 2010, том 162, номер 3, 422-438

DOI: https://doi.org/10.4213/tmf6480

Использование Общероссийского математического портала Math-Net.Ru подразумевает, что вы прочитали и согласны с пользовательским соглашением http://www . mathnet.ru/rus/agreement

Параметры загрузки:

IP : 18.208 .226 .222

26 апреля 2023 г., 11:48:39

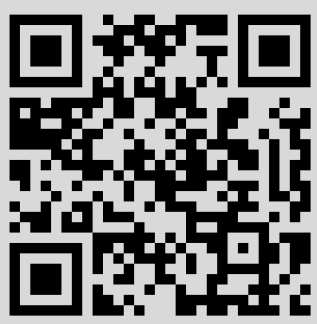




\title{
ФИЗИКА
}

Том 162, № 3

март, 2010

2010 г.

Т. В. Рябуха*

\section{О ФУНКЦИОНАЛАХ ДЛЯ СРЕДНИХ ЗНАЧЕНИЙ НАБЛЮДАЕМЫХ ОДНОМЕРНЫХ БЕСКОНЕЧНЫХ СИСТЕМ ЧАСТИЦ}

\begin{abstract}
Изучается проблема существования средних значений наблюдаемых для систем бесконечного числа частиц. С использованием решения задач Коши иерархий Боголюбова-Борна-Грина-Кирквуда-Ивона и двойственной к ней доказано локальное по времени существование функционалов для средних значений в случаях, когда или наблюдаемые, или состояния изменяются со временем. Также обсуждается проблема существования таких функционалов для нескольких различных классов наблюдаемых и для произвольного временно́го интервала.
\end{abstract}

Ключевые слова: система бесконечного числа частиц, иерархия Боголюбова-БорнаГрина-Кирквуда-Ивона, двойственная иерархия Боголюбова-Борна-Грина-КирквудаИвона, кумулянт (семиинвариант), среднее значение наблюдаемых.

\section{1. ВВЕДЕНИЕ}

В последнее десятилетие был достигнут значительный прогресс в изучении динамики бесконечного числа частиц [1]-[4]. Как хорошо известно, эволюция состояний таких систем полностью определяется задачей Коши для иерархии Боголюбова-Борна-Грина-Кирквуда-Ивона (ББГКИ) [1], [5]. Решение этой задачи в виде разложения в ряд, полученный в результате интегрирования ряда возмущений по временны́м переменным, было впервые построено в работе [6] для одномерной системы частиц, взаимодействующих посредством короткодействующего потенциала с твердой сердцевиной, при начальных условиях, близких к равновесным состояниям. Расходимость интегралов по конфигурационным переменным в каждом члене разложения является типичной проблемой, усложняющей построение решения для системы бесконечного числа частиц. В методе области взаимодействия [7] (см. также [1], [6]) предлагается один из подходов, с помощью которого можно обойти это препятствие. Еще один результат о динамике бесконечного числа частиц для системы трехмерных упругих шаров основывается на построении решения иерархии ББГКИ в виде ряда по возмущениям [2], [4], [8], [9] .

* Институт математики НАН Украины, Киев, Украина. E-mail: vyrtum@imath.kiev.ua 
Недавно в работе [10] было получено представление решения задачи Коши для иерархии ББГКИ в виде разложения по кластерам (группам) частиц, эволюция которых определяется в терминах кумулянта соответствующего порядка операторов эволюции конечного числа частиц. Вышеупомянутые представления решения иерархии ББГКИ являются частными случаями предложенного в [10]. Метод области взаимодействия для этого представления разработан в статье [11].

В настоящей статье развивается подобный подход регуляризации к решению двойственной иерархии ББГКИ [12], описывающей эволюцию маргинальных наблюдаемых многокомпонентных систем [12], [13]. Доказывается существование функционалов для средних значений систем бесконечного числа частиц как в случаях, когда рассматривается эволюция наблюдаемых, так и когда рассматривается эволюция состояний.

Сначала приведем некоторые факты, касающиеся описания систем бесконечного числа частиц. Рассмотрим одномерную систему тождественных частиц (единичной массы), взаимодействующих через короткодействующий парный потенциал $\Phi$ (с твердой сердцевиной), обладающий следующими свойствами:

$$
\begin{gathered}
\Phi \in C^{2}([\sigma, R]), \quad 0<\sigma<R<\infty, \\
\Phi(|q|)=\left\{\begin{array}{ll}
+\infty, & |q| \in[0, \sigma), \\
0, & |q| \in(R, \infty),
\end{array}, \quad \Phi^{\prime}(\sigma+0)=0,\right.
\end{gathered}
$$

где $R$ - радиус взаимодействия частиц, $\sigma>0$ - длина частицы (упругого стержня).

Пусть $i$-я частица характеризуется координатой $q_{i}$ центра частицы и импульсом $p_{i}$. Положим $x_{i} \equiv\left(q_{i}, p_{i}\right) \in \mathbb{R} \times \mathbb{R}, i \geqslant 1$. Для системы $n$ частиц неравенства

$$
\left|q_{i}-q_{j}\right| \geqslant \sigma, \quad i, j \in\{1, \ldots, n\}, \quad i \neq j, \quad n \geqslant 2
$$

определяют допустимые конфигурации. Область $W_{n}$ запрещенных конфигураций это множество координат $\left\{q_{1}, \ldots, q_{n}\right\} \in \mathbb{R}^{n}, n \geqslant 2$, таких, что по крайней мере для одной пары индексов $i, j \in\{1, \ldots, n\}, i \neq j$, выполнено неравенство $\left|q_{i}-q_{j}\right|<\sigma$.

В начальный момент времени $t=0$ наблюдаемая описывается последовательностью маргинальных (s-частичных) функций $G(0)=\left(G_{0}, G_{1}\left(0, x_{1}\right), \ldots, G_{s}\left(0, x_{1}, \ldots\right.\right.$ $\left.\left.\ldots, x_{s}\right), \ldots\right)$, а состояние - последовательностью маргинальных ( $s$-частичных) функций распределения $F(0)=\left(F_{0}, F_{1}\left(0, x_{1}\right), \ldots, F_{s}\left(0, x_{1}, \ldots, x_{s}\right), \ldots\right)$. Среднее значение (математическое ожидание) наблюдаемой $G(0)$ для системы в состоянии $F(0)$ определяется следующим функционалом [1], [13]:

$$
\begin{aligned}
\langle G\rangle(0) & =\langle G(0) \mid F(0)\rangle= \\
& =\sum_{s=0}^{\infty} \frac{1}{s !} \int_{\left(\mathbb{R}^{s} \backslash W_{s}\right) \times \mathbb{R}^{s}} d x_{1} \ldots d x_{s} G_{s}\left(0, x_{1}, \ldots, x_{s}\right) F_{s}\left(0, x_{1}, \ldots, x_{s}\right),
\end{aligned}
$$

где $F_{0}=1, G_{0}=0$.

Введем пространство $C_{\gamma}$ последовательностей $g=\left(g_{0}, g_{1}\left(x_{1}\right), \ldots, g_{n}\left(x_{1}, \ldots, x_{n}\right), \ldots\right)$ ограниченных (непрерывных) функций $g_{n}\left(x_{1}, \ldots, x_{n}\right), n \geqslant 0, g_{0}$ - число, заданных на фазовом пространстве $\left(\mathbb{R}^{n} \backslash W_{n}\right) \times \mathbb{R}^{n}$, симметричных относительно любых перестановок аргументов $x_{i}, i=1, \ldots, n$, и равных нулю в области $W_{n}$ запрещенных 
конфигураций, с нормой [13]

$$
\|g\|_{C \gamma}=\sup _{n \geqslant 0} \frac{\gamma^{n}}{n !} \sup _{x_{1}, \ldots, x_{n}}\left|g_{n}\left(x_{1}, \ldots, x_{n}\right)\right|,
$$

где $0<\gamma<1$ - некоторая константа. Обозначим через $C_{\gamma, 0}$ подпространство в $C_{\gamma}$, элементами которого являются конечные последовательности непрерывно дифференцируемых функций с компактным носителем в конфигурационном пространстве. Последовательность функций $g \in C_{\gamma, 0}$ трактуется как квазинаблюдаемая (аналог локальной наблюдаемой [1], [14]).

Состояния систем бесконечного числа частиц задаются как элементы пространства $L_{\xi, \beta}^{\infty}$ последовательностей $f=\left(f_{0}, f_{1}\left(x_{1}\right), \ldots, f_{n}\left(x_{1}, \ldots, x_{n}\right), \ldots\right)$ функций $f_{n}\left(x_{1}, \ldots, x_{n}\right), n \geqslant 0, f_{0}=1$, заданных на фазовом пространстве $\left(\mathbb{R}^{n} \backslash W_{n}\right) \times \mathbb{R}^{n}$, симметричных относительно произвольных перестановок аргументов $x_{i}, i=1, \ldots, n$, и равных нулю на запрещенных конфигурациях $W_{n}$, с нормой [1], [6]

$$
\|f\|_{L_{\xi, \beta}^{\infty}}=\sup _{n \geqslant 0} \xi^{-n} \sup _{x_{1}, \ldots, x_{n}}\left|f_{n}\left(x_{1}, \ldots, x_{n}\right)\right| \exp \left\{\beta \sum_{i=1}^{n} \frac{p_{i}^{2}}{2}\right\},
$$

где $\xi, \beta>0$ - некоторые константы.

Заметим, что если $F(0) \in L_{\xi, \beta}^{\infty}$ и $G(0) \in C_{\gamma, 0}$, то имеет место следующая оценка:

$$
|\langle G(0) \mid F(0)\rangle| \leqslant\|G(0)\|_{C_{\gamma}}\|F(0)\|_{L_{\xi, \beta}^{\infty}} \sum_{s=0}^{\infty}\left(\frac{C \xi}{\gamma} \sqrt{\frac{2 \pi}{\beta}}\right)^{s},
$$

поэтому функционал (1.2) корректно определен при условии

$$
\xi<\frac{\gamma}{C} \sqrt{\frac{\beta}{2 \pi}}, \quad C=\max _{i=1, \ldots, s}\left|l_{i}(0)\right|,
$$

где $\left|l_{i}(0)\right|$ - длина интервала $l_{i}(0)$ такого, что множество

$$
\Omega_{s}(0)=l_{1}(0) \times \cdots \times l_{s}(0)
$$

является носителем функции $G_{s}(0)$ в конфигурационном пространстве.

Для наблюдаемой аддитивного типа $G^{(1)}(0)=\left(0, a_{1}\left(0, x_{1}\right), 0, \ldots, 0, \ldots\right)$ функционал (1.2) имеет вид

$$
\left\langle G^{(1)}\right\rangle(0)=\left\langle G^{(1)}(0) \mid F(0)\right\rangle=\int_{\mathbb{R} \times \mathbb{R}} d x_{1} a_{1}\left(0, x_{1}\right) F_{1}\left(0, x_{1}\right) .
$$

Если $F(0) \in L_{\xi, \beta}^{\infty}$ и $G(0) \in C_{\gamma, 0}$, то данный функционал корректно определен для любого значения параметра $\xi>0$, поскольку

$$
\left|\left\langle G^{(1)}(0) \mid F(0)\right\rangle\right| \leqslant\left\|G^{(1)}(0)\right\|_{C_{\gamma}}\|F(0)\|_{L_{\xi, \beta}^{\infty}} \frac{C \xi}{\gamma} \sqrt{\frac{2 \pi}{\beta}}<\infty .
$$

В произвольный момент времени $t \in \mathbb{R}$ среднее значение наблюдаемой определяется следующим функционалом [1]:

$$
\begin{aligned}
\langle G\rangle(t) & =\langle G(t) \mid F(0)\rangle= \\
& =\sum_{s=0}^{\infty} \frac{1}{s !} \int_{\left(\mathbb{R}^{s} \backslash W_{s}\right) \times \mathbb{R}^{s}} d x_{1} \ldots d x_{s} G_{s}\left(t, x_{1}, \ldots, x_{s}\right) F_{s}\left(0, x_{1}, \ldots, x_{s}\right),
\end{aligned}
$$


где $G(t)=\left(0, G_{1}\left(t, x_{1}\right), \ldots, G_{s}\left(t, x_{1}, \ldots, x_{s}\right), \ldots\right)$ - решение задачи Коши для двойственной иерархии ББГКИ [12], [13] с начальными условиями $G(0)$, или таким функционалом:

$$
\begin{aligned}
\langle G\rangle(t) & =\langle G(0) \mid F(t)\rangle= \\
& =\sum_{s=0}^{\infty} \frac{1}{s !} \int_{\left(\mathbb{R}^{s} \backslash W_{s}\right) \times \mathbb{R}^{s}} d x_{1} \ldots d x_{s} G_{s}\left(0, x_{1}, \ldots, x_{s}\right) F_{s}\left(t, x_{1}, \ldots, x_{s}\right),
\end{aligned}
$$

где $F(t)=\left(1, F_{1}\left(t, x_{1}\right), \ldots, F_{s}\left(t, x_{1}, \ldots, x_{s}\right), \ldots\right)$ - решение задачи Коши для иерархии ББГКИ [1], [3], [14] с начальными условиями $F(0)$.

Опишем кратко структуру статьи. В разделе 2 процедура регуляризации, предложенная в работе [11], применена к решению двойственной иерархии ББГКИ. Затем в разделах 3 и 4 доказано существование функционалов (1.5) и (1.6) с использованием результатов работы [11]. В разделе 5 приведены некоторые заключительные замечания.

\section{2. РЕГУЛЯРИЗОВАННОЕ РЕШЕНИЕ ДВОЙСТВЕННОЙ ИЕРАРХИИ ББГКИ}

Перед тем как доказать существование функционала (1.5), сформулируем некоторые факты, касающиеся решения двойственной иерархии ББГКИ, и построим регуляризованное представление решения, которое в следующем разделе позволит компенсировать расходящиеся члены, возникающие в функционале (1.5) в случае систем бесконечного числа частиц.

Пусть

$$
\left\{x_{1}, \ldots, x_{s}\right\}=Y, \quad\left\{x_{j_{1}}, \ldots, x_{j_{s-n}}\right\}=Y \backslash X,
$$

где $\left\{j_{1}, \ldots, j_{s-n}\right\} \subseteq\{1, \ldots, s\}$, т.е.

$$
X=\left\{x_{1}, \stackrel{j_{1}}{\vee}, \ldots, \stackrel{j_{s}-n}{\vee}, x_{s}\right\}, \quad\left\{x_{1}, \stackrel{j_{k}}{\vee}, x_{s}\right\} \equiv\left\{x_{1}, \ldots, x_{j_{k-1}}, x_{j_{k+1}}, \ldots, x_{s}\right\} .
$$

Обозначим через $|X|$ количество элементов в множестве $X, 0 \leqslant|X|=n \leqslant s$. Поскольку функции, рассматриваемые в этой статье, симметричны относительно перестановок аргументов, можно считать, что элементы фазового пространства множества (а не упорядоченные наборы) точек.

Введем оператор эволюции $S_{s}(t, Y), s \geqslant 1$, определенный на пространстве $C_{\gamma}$ следующей формулой [1]:

$$
\begin{aligned}
& S_{|Y|}(t, Y) f_{|Y|}(Y)= \\
& \quad= \begin{cases}f_{|Y|}\left(\mathbf{X}_{1}(t, Y), \ldots, \mathbf{X}_{|Y|}(t, Y)\right), & Y \in\left(\left(\mathbb{R}^{|Y|} \backslash W_{|Y|}\right) \times \mathbb{R}^{|Y|}\right) \backslash \mathcal{M}_{|Y|}^{0}, \\
0, & Y \in\left(W_{|Y|} \times \mathbb{R}^{|Y|}\right) \cup \mathcal{M}_{|Y|}^{0},\end{cases}
\end{aligned}
$$

где $\mathbf{X}_{j}(t, Y)$ - фазовая траектория [1] системы из $|Y|=s$ частиц с начальными условиями $\mathbf{X}_{j}(0, Y)=x_{j}, j=1, \ldots,|Y|$, и $S_{|Y|}(0)=I$ - единичный оператор. Заметим, что фазовая траектория системы с потенциалом взаимодействия (1.1) 
определена не для любых начальных условий $\left\{x_{1}, \ldots, x_{n}\right\} \in\left(\mathbb{R}^{n} \backslash W_{n}\right) \times \mathbb{R}^{n}$, а почти всюду на фазовом пространстве $\left(\mathbb{R}^{n} \backslash W_{n}\right) \times \mathbb{R}^{n}$, а именно вне определенного множества $\mathcal{M}_{n}^{0}$ нулевой меры Лебега. Множество $\mathcal{M}_{n}^{0}$ содержит начальные условия $\left\{x_{1}, \ldots, x_{n}\right\} \in\left(\mathbb{R}^{n} \backslash W_{n}\right) \times \mathbb{R}^{n}$, для которых, во-первых, в момент времени $t \in(-\infty,+\infty)$ происходят кратные столкновения (тройные и т.д.), во-вторых, за конечный промежуток времени происходит бесконечное число столкновений [1], [9]. Оператор эволюции (2.1) корректно определен при $t \in(-\infty,+\infty)$ при выполнении условий (1.1) на потенциал взаимодействия $\Phi$ [1].

Для начальных условий $G(0) \in C_{\gamma}$ в случае $\gamma<e^{-1}$ решение задачи Коши для двойственной иерархии ББГКИ задается формулой [12]

$$
G_{|Y|}(t, Y)=\sum_{n=0}^{s} \sum_{1=j_{1}<\cdots<j_{s-n}}^{s} \mathfrak{A}_{|X|+1}(t,\{Y \backslash X\}, X) G_{|Y \backslash X|}(0, Y \backslash X),
$$

где $0 \leqslant|X|=n \leqslant s$. В этой формуле оператор $\mathfrak{A}_{|X|+1}(t,\{Y \backslash X\}, X)$ - это кумулянт $(|X|+1)$-го порядка для операторов эволюции $S_{\left|Y_{i}\right|}\left(t, Y_{i}\right)(2.1),\left|Y_{i}\right| \geqslant 1$, который определяется следующим образом [10]:

$$
\mathfrak{A}_{|X|+1}(t,\{Y \backslash X\}, X)=\sum_{\mathbf{P}:\{Y \backslash X\} \cup X=\cup_{i} Y_{i}}(-1)^{|\mathbf{P}|-1}(|\mathbf{P}|-1) ! \prod_{Y_{i} \subset \mathbf{P}} S_{\left|Y_{i}\right|}\left(t, Y_{i}\right),
$$

где сумма берется по всем возможным разбиениям $\mathbf{P}$ множества $\widehat{Y} \equiv\{Y \backslash X\} \cup X$ на $|\mathbf{P}|$ непустых попарно непересекающихся подмножеств $Y_{i} \subset \widehat{Y}, Y_{i} \cap Y_{j}=\varnothing$, $i \neq j$, причем $\{Y \backslash X\}$ означает, что $Y \backslash X$ является кластером взаимодействующих между собой частиц и трактуется как один элемент множества $\widehat{Y}$, т.е. в этом случае $|\widehat{Y}|=|X|+1=n+1$. Положим $\mathfrak{A}_{2}(t,\{0\},\{Y\})=\mathfrak{A}_{1}\left(t_{1},\{Y\}\right)$. Например, кумулянты первого и второго порядков имеют соответственно вид

$$
\begin{aligned}
\mathfrak{A}_{1}(t,\{Y\}) & =S_{s}(t, Y), \\
\mathfrak{A}_{2}\left(t,\left\{Y \backslash x_{s}\right\}, x_{s}\right) & =S_{s}(t, Y)-S_{s-1}\left(t, Y \backslash x_{s}\right) S_{1}\left(t, x_{s}\right),
\end{aligned}
$$

где $\{Y\}$ и $\left\{Y \backslash x_{s}\right\}$ соответственно трактуются как один элемент при разбиении множества $\widehat{Y}$ на подмножества ${ }^{1)}$.

Заметим, что кумулянт можно определить на множестве, элементами которого являются многокомпонентные непересекающиеся множества, состоящие из конечного числа частиц. Например, кумулянт второго порядка, определенный на двухэлементном множестве, элементы которого - непересекающиеся множества $Y \backslash X$ и $Z$, имеет вид

$$
\mathfrak{A}_{2}(t,\{Y \backslash X\},\{Z\})=S_{|Y \backslash X|+|Z|}(t, Y \backslash X, Z)-S_{|Y \backslash X|}(t, Y \backslash X) S_{|Z|}(t, Z) .
$$

\footnotetext{
1) Кумулянт - это эволюционный оператор, физическая интерпретация которого еще четко не определена, он характеризует взаимодействие между кластерами (группами) частиц. И таким кластером может быть как одна частица, так и целое множество частиц, между которыми происходит взаимодействие. Поэтому фактически порядок кумулянта определяется количеством интересующих нас кластеров и указывается как нижний индекс. И в том случае, когда кластер - это некое неделимое множество, то мы заключаем его (множество) в фигурные скобки.
} 
Лемма 2.1 [11]. Для кумулянта $(n+1)$-го порядка $\mathfrak{A}_{|X|+1}(t,\{Y \backslash X\}, X)$ в формуле (2.3) имеет место следующее представление:

$$
\begin{aligned}
\mathfrak{A}_{|X|+1}(t,\{Y \backslash X\}, X)= & \sum_{\substack{Z \subset X, Z \neq \varnothing}} \mathfrak{A}_{2}(t,\{Y \backslash X\},\{Z\}) \sum_{\mathbf{Q}: X \backslash Z=\bigcup_{l} X_{l}}(-1)^{|\mathbf{Q}|}|\mathbf{Q}| ! \times \\
& \times \prod_{X_{l} \subset \mathbf{Q}} \mathfrak{A}_{1}\left(t,\left\{X_{l}\right\}\right), \quad 1 \leqslant|X|=n \leqslant|Y|=s,
\end{aligned}
$$

где первая сумма берется по всем непустым подмножествам $Z$ множества $X$, а вторая - по всем разбиениям $\mathbf{Q}$ множества $X \backslash Z$ на $|\mathbf{Q}|$ непустых непересекающихся подмножеств $X_{l} \subset X \backslash Z, X_{k} \cap X_{l}=\varnothing, k \neq l$.

Применим данную лемму при построении нового представления для решения задачи Коши двойственной иерархии ББГКИ.

Принимая во внимание представление (2.6) для решения (2.2), можно доказать, что функция $G_{|Y \backslash X|}(0, Y \backslash X)$ не зависит от множества переменных $X_{l} \subset X \backslash Z$. Поскольку $X_{l} \not \subset Y \backslash X$, кумулянты первого порядка $\mathfrak{A}_{1}\left(t,\left\{X_{l}\right\}\right)$ не действуют на переменные функции $G_{|Y \backslash X|}(0, Y \backslash X)$. Поэтому эквивалентным соотношению $(2.2)$ будет следующее:

$$
\begin{aligned}
G_{|Y|}(t, Y)= & \mathfrak{A}_{1}(t,\{Y\}) G_{|Y|}(0, Y)+ \\
& +\sum_{n=1}^{s} \sum_{1=j_{1}<\cdots<j_{s-n}}^{s} \sum_{\substack{Z \subset X, Z \neq \varnothing}} \mathfrak{A}_{2}(t,\{Y \backslash X\},\{Z\}) G_{|Y \backslash X|}(0, Y \backslash X) \times \\
& \times \sum_{\mathbf{Q}: X \backslash Z=\cup_{l} X_{l}}(-1)^{|\mathbf{Q}|}|\mathbf{Q}| !, \quad 1 \leqslant|X|=n \leqslant s .
\end{aligned}
$$

Отсюда в силу равенств

$$
\sum_{\mathbf{Q}: X \backslash Z=\bigcup_{l} X_{l}}(-1)^{|\mathbf{Q}|}|\mathbf{Q}| !=\sum_{k=1}^{|X \backslash Z|}(-1)^{k} k ! s(|X \backslash Z|, k)
$$

И

$$
\sum_{k=1}^{m}(-1)^{k} k ! s(m, k)=(-1)^{m}, \quad m \geqslant 1,
$$

где $s(|X \backslash Z|, k) \equiv s(m, k)$ - числа Стирлинга второго рода, вытекает, что эквивалентным представлением решения (2.2) задачи Коши двойственной иерархии ББГКИ является следующее разложение (регуляризованное решение):

$$
\begin{aligned}
& G_{|Y|}(t, Y)=\mathfrak{A}_{1}(t,\{Y\}) G_{|Y|}(0, Y)+
\end{aligned}
$$

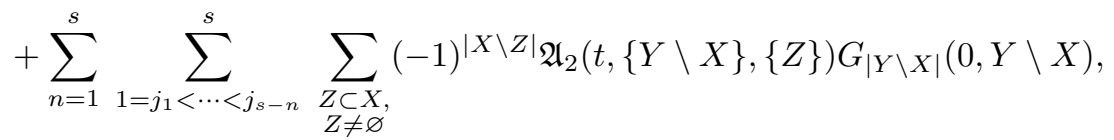

где $1 \leqslant|X|=n \leqslant|Y|=s$. 
Таким образом, для $G(0) \in C_{\gamma}$ кумулянтное представление $(2.2),(2.3)$ решения задачи Коши двойственной иерархии ББГКИ эквивалентно регуляризованному решению (2.7), структура которого позволяет компенсировать расходящиеся члены, возникающие в функционале (1.5) при $F(0) \in L_{\xi, \beta}^{\infty}, G(0) \in C_{\gamma, 0}$.

ЛЕмма 2.2. Если $G(0) \in C_{\gamma}$, то при условии $0<\gamma<1$ для разложения (2.7) имеет место оценка

$$
\left|G_{|Y|}(t, Y)\right| \leqslant 2 e^{2}\|G(0)\|_{C_{\gamma}} \frac{s !}{\gamma^{s}} .
$$

ДоказАтельство. Пусть $G(0) \in C_{\gamma}$. Согласно формулам (2.1) и (2.4) соответственно выполняются следующие неравенства:

$$
\begin{gathered}
\left|\mathfrak{A}_{1}(t,\{Y\}) G_{|Y|}(0, Y)\right| \leqslant\|G(0)\|_{C_{\gamma}} \frac{|Y| !}{\gamma^{|Y|}}, \\
\left|\mathfrak{A}_{2}(t,\{Y \backslash X\},\{Z\}) G_{|Y \backslash X|}(0, Y \backslash X)\right| \leqslant 2\|G(0)\|_{C_{\gamma}} \frac{|Y \backslash X| !}{\gamma^{|Y \backslash X|}} .
\end{gathered}
$$

Из этих неравенств получаем следующую оценку для разложения (2.7):

$$
\left|G_{|Y|}(t, Y)\right| \leqslant 2\|G(0)\|_{C_{\gamma}} \sum_{n=0}^{s} \sum_{1=j_{1}<\cdots<j_{s-n}}^{s} 2^{n} \frac{(s-n) !}{\gamma^{s-n}} .
$$

Поскольку

$$
0<\gamma<1, \quad \sum_{1=j_{1}<\cdots<j_{s-n}}^{s} 1=\frac{s !}{(s-n) ! n !}, \quad \sum_{n=0}^{s} \frac{2^{n}}{n !} \leqslant e^{2},
$$

оценка (2.11) принимает вид (2.8), указанный в утверждении леммы.

\section{3. СРЕДНЕЕ ЗНАЧЕНИЕ НАБЛЮДАЕМЫХ: ЭВОЛЮЦИЯ НАБЛЮДАЕМЫХ}

Установим существование функционала (1.5) в случае эволюции наблюдаемых, которые определяются регуляризованным разложением (2.7).

УтВЕРЖДЕНИЕ 3.1. Для системы частии, с парным потенииалом взаимодействия $\Phi$, удовлетворяюшим условиям (1.1), если $F(0) \in L_{\xi, \beta}^{\infty}, G(0) \in C_{\gamma, 0}$ и наблюдаемая $G(t)$ определяется разложением (2.7), то при

$$
\xi<\frac{\gamma}{e \max \left(C ; 2 \widetilde{C}_{1}\right)} \sqrt{\frac{\beta^{\prime \prime}}{2 \pi}}, \quad 0 \leqslant t<t_{0}, \quad t_{0} \equiv \frac{1}{\widetilde{C}_{2}}\left(-\widetilde{C}_{1}+\frac{\gamma}{2 e \xi} \sqrt{\frac{\beta^{\prime \prime}}{2 \pi}}\right),
$$

функиионал (1.5) корректно определен и выполняется следующая оценка:

$$
\begin{aligned}
|\langle G(t) \mid F(0)\rangle| \leqslant & 2 e^{C}\|F(0)\|_{L_{\xi, \beta}^{\infty}}\|G(0)\|_{C_{\gamma}} \sum_{s=0}^{\infty}\left(\frac{e C \xi}{\gamma} \sqrt{\frac{2 \pi}{\beta^{\prime \prime}}}\right)^{s} \times \\
& \times \sum_{n=0}^{\infty}\left(\frac{2 e \xi}{\gamma} \sqrt{\frac{2 \pi}{\beta^{\prime \prime}}}\right)^{n}\left(\widetilde{C}_{1}+\widetilde{C}_{2} t\right)^{n} .
\end{aligned}
$$


Здесъ $C=\max _{i=j_{1}, \ldots, j_{s-n}}\left|l_{i}(0)\right| u\left|l_{i}(0)\right|$ - длина интервала $l_{i}(0)$ из компакта $\Omega_{|Y \backslash X|}(0)=l_{j_{1}}(0) \times \cdots \times l_{j_{|Y \backslash X|}}(0)$, в котором лежит носитель функиии $G_{s-n}(0)$, а константы задаются равенствами

$$
\begin{gathered}
\widetilde{C}_{1}=\max (2 R, 1), \quad \widetilde{C}_{2}=\max \left(2(4 b+1), \frac{2}{\beta^{\prime}}\right), \\
\beta=\beta^{\prime}+\beta^{\prime \prime}, \quad b \equiv \sup _{q \in[\sigma, R]}|\Phi(q)|\left(\left[\frac{R}{\sigma}\right]\right),
\end{gathered}
$$

$[R / \sigma]$ - иелая часть числа $R / \sigma$.

ДокАЗАтЕЛЬСтво. Пусть $F(0) \in L_{\xi, \beta}^{\infty}, G(0) \in C_{\gamma, 0}$, при этом наблюдаемая $G_{|Y \backslash X|}(0, Y \backslash X)$ имеет носителем в конфигурационном пространстве компактное множество, которое мы будем обозначать через $\Omega_{|Y \backslash X|}(0)=l_{j_{1}}(0) \times \cdots \times l_{j_{|Y \backslash X|}}(0)$, где $l_{i}(0)$ - такой сегмент, что $q_{i} \in l_{i}(0), i=j_{1}, \ldots, j_{s-n}$. Если $n=0$, то компакт это $\Omega_{|Y|}(0)=l_{1}(0) \times \cdots \times l_{|Y|}(0)($ см. $(1.3))$.

Если $G_{s}\left(t, x_{1}, \ldots, x_{s}\right) \equiv G_{|Y|}(t, Y)$ - решение двойственной иерархии ББГКИ [12], определенное разложением (2.7), то выражение для функционала (1.5) имеет вид

$$
\begin{aligned}
& \langle G(t) \mid F(0)\rangle=\sum_{s=0}^{\infty} \frac{1}{s !} \int_{\left(\mathbb{R}^{s} \backslash W_{s}\right) \times \mathbb{R}^{s}} d Y\left(\mathfrak{A}_{1}(t,\{Y\}) G_{|Y|}(0, Y)+\right. \\
& +\sum_{n=1}^{s} \sum_{1=j_{1}<\cdots<j_{s-n}}^{s} \sum_{\substack{Z \subset X, Z \neq \varnothing}}(-1)^{\left.|X \backslash Z|_{\mathfrak{A}_{2}}(t,\{Y \backslash X\},\{Z\}) G_{|Y \backslash X|}(0, Y \backslash X)\right) \times} \\
& \quad \times F_{|Y|}(0, Y), \quad 1 \leqslant|X|=n \leqslant|Y|=s .
\end{aligned}
$$

В данном выражении область $\mathbb{R}^{s} \backslash W_{s}$ интегрирования по конфигурационным переменным ограничивается до множества, на котором подынтегральное выражение конечно и отлично от нуля. Поскольку кумулянт первого порядка $\mathfrak{A}_{1}(t,\{Y\})$ - это оператор $S_{|Y|}(t, Y)(2.1)$, в разложении (3.3) функция $\mathfrak{A}_{1}(t,\{Y\}) G_{|Y|}(0, Y) F_{|Y|}(0, Y)$ интегрируется не по всему конфигурационному пространству, а только по переменным такого компакта $\Omega_{|Y|}(0) \subset \mathbb{R}^{s} \backslash W_{s}$, который при интегрировании сдвигается вдоль конфигурационной траектории и имеет конечный объем $V_{\Omega_{|Y|}(0)}=\Pi_{i=1}^{|Y|}\left|l_{i}(0)\right|$.

Выражение

$$
\sum_{n=1}^{s} \sum_{1=j_{1}<\cdots<j_{s-n}}^{s} \sum_{\substack{Z \subset X, Z \neq \varnothing}}(-1)^{|X \backslash Z|} \mathfrak{A}_{2}(t,\{Y \backslash X\},\{Z\}) G_{|Y \backslash X|}(0, Y \backslash X) F_{|Y|}(0, Y)
$$

равно нулю, если за временно́й интервал $[0, t)$ частицы с произвольными начальными условиями $X$ не взаимодействуют с частицами с фиксированными начальными условиями $Y \backslash X$, поскольку в этом случае для кумулянта второго порядка $\mathfrak{A}_{2}(t,\{Y \backslash X\},\{Z\})(2.5)$ при $Z \subset X, Z \neq \varnothing$, выполняется следующее свойство:

$$
\mathfrak{A}_{2}(t,\{Y \backslash X\},\{Z\}) G_{|Y \backslash X|}(0, Y \backslash X)=0,
$$


которое будем называть свойством невзаимодействия. Из-за конечности потенциала $\Phi$, удовлетворяющего условиям (1.1), во временно́м интервале $[0, t)$ не может произойти взаимодействие (упругое столкновение) между частицами на расстоянии, большем, чем могут преодолеть эти частицы в совокупности. Благодаря этому в разложении (3.3) область интегрирования по конфигурационным переменным ограничивается областью $\Omega_{|Y|}\left(t, \Omega_{|Y \backslash X|}(0)\right) \equiv \Omega_{|Y \backslash X|}(0) \times \Omega_{|X|}(t)$, где $\Omega_{|X|}(t)$ - область взаимодействия частиц с произвольными начальными условиями $X$ с частицами с фиксированными начальными условиями $Y \backslash X, Y \backslash X \in \Omega_{|Y \backslash X|}(0)$, во временно́м интервале $[0, t)$; такая область интегрирования имеет конечный объем $V_{\Omega_{|Y|}(t)}$.

Действительно, в начальный момент $t=0 i$-я частица с фазовой координатой из $Y \backslash X, i=j_{1}, \ldots, j_{|Y \backslash X|}$, имеет фиксированные значения импульса $p_{i}$ и локализованной конфигурационной координаты $q_{i}$. Получается, что в произвольный момент времени $\tau, \tau \in[0, t)$, импульс $p_{i}(\tau, Y \backslash X)$ определяется из задачи Коши уравнений Гамильтона [1] для системы конечного числа $|Y \backslash X|$ частиц. Поэтому за время $t$ такая частица преодолевает расстояние $\int_{0}^{t} d \tau\left|p_{i}(\tau, Y \backslash X)\right|$. В целом имеем, что $|Y \backslash X|=s-n$ частиц и $|Y|=s$ частиц преодолевают расстояния

$$
\sum_{x_{i} \in Y \backslash X} \int_{0}^{t} d \tau\left|p_{i}(\tau, Y \backslash X)\right| \quad \text { и } \quad \sum_{i=1}^{s} \int_{0}^{t} d \tau\left|p_{i}(\tau, Y)\right|
$$

соответственно. Предположим, что интервал $l_{|Y \backslash X|}(0)$ таков, что $\left|l_{|Y \backslash X|}(0)\right|=$ $\max _{i=j_{1}, \ldots, j_{|Y \backslash X|}}\left|l_{i}(0)\right|$. Приняв во внимание радиус взаимодействия $R$, последовательно откладываем $|Y|$ отрезков длины $R+\int_{0}^{t} d \tau\left|p_{i}(\tau, Y)\right|, i=j_{1}, \ldots, j_{s}$, слева и справа от интервала $l_{|Y \backslash X|}(0)$. Получим интервал, который мы обозначим через $l_{|X|}(t)$, такой, что

$$
\underbrace{l_{|X|}(t) \times \cdots \times l_{|X|}(t)}_{n} \times \Omega_{|Y \backslash X|}(0)=\Omega_{|Y|}\left(t, \Omega_{|Y \backslash X|}(0)\right),
$$

и его длина оценивается следующим образом:

$$
\left|l_{|X|}(t)\right| \leqslant\left|l_{|Y \backslash X|}(0)\right|+2 s R+2 \sum_{i=1}^{s} \int_{0}^{t} d \tau\left|p_{i}(\tau, Y)\right|,
$$

т.е. область взаимодействия $\Omega_{|X|}(t)$ определяется как $\underbrace{\Omega_{|X|}(t)=l_{|X|}(t) \times \cdots \times l_{|X|}(t)}_{n}$. Заметим, что для любого $Z \subset X$ справедливо включение $\Omega_{|Z|}(t) \subset \Omega_{|X|}(t)$.

Для парного потенциала взаимодействия $\Phi$, удовлетворяющего условиям (1.1), выполняется следующее неравенство:

$$
\left|\sum_{\substack{i, j=1, i<j}}^{s} \Phi\left(q_{i}-q_{j}\right)\right| \leqslant b s, \quad b \equiv \sup _{q \in[\sigma, R]}|\Phi(q)|\left(\left[\frac{R}{\sigma}\right]\right) .
$$


Вследствие неравенства $2\left|p_{i}(\tau)\right| \leqslant p_{i}^{2}(\tau)+1$ для произвольного значения импульса $p_{i}(\tau) \equiv p_{i}(\tau, Y)$ из закона сохранения энергии

$$
\sum_{i=1}^{s} \frac{p_{i}^{2}}{2}+\sum_{\substack{i, j=1, i<j}}^{s} \Phi\left(q_{i}-q_{j}\right)=\sum_{i=1}^{s} \frac{p_{i}^{2}(\tau)}{2}+\sum_{\substack{i, j=1, i<j}}^{s} \Phi\left(q_{i}(\tau)-q_{j}(\tau)\right)
$$

и условий (3.6) следует ограниченность сверху суммы импульсов в момент времени $\tau$ :

$$
2 \sum_{i=1}^{s} p_{i}(\tau) \leqslant \sum_{i=1}^{s} p_{i}^{2}+(4 b+1) s
$$

где $b$ определяется в (3.6). Принимая во внимание (3.7), из (3.5) можно вывести неравенство

$$
\left|l_{|X|}(t)\right| \leqslant\left|l_{|Y \backslash X|}(0)\right|+2 s(R+(4 b+1) t)+t \sum_{i=1}^{s} p_{i}^{2} .
$$

Пусть

$$
C=\max _{i=j_{1}, \ldots, j_{s-n}}\left|l_{i}(0)\right|, \quad C_{1} \equiv 2 R, \quad C_{2} \equiv 2(4 b+1),
$$

тогда объем $V_{\Omega_{|Y|}(t)}$ области интегрирования $\Omega_{|Y|}\left(t, \Omega_{|Y \backslash X|}(0)\right)$ конечен:

$$
V_{\Omega_{|Y|}(t)} \leqslant\left(C+\left(C_{1}+C_{2} t\right) s+t \sum_{i=1}^{s} p_{i}^{2}\right)^{n} C^{s-n} .
$$

Таким образом, равенство (3.4) выполняется в $s$-м члене ряда (3.3) при интегрировании по конфигурационным переменным вне области взаимодействия $\Omega_{|X|}(t)$.

Учитывая свойство невзаимодействия (3.4), из леммы 2.2 и неравенства (3.9) получим, что для функционала (3.3) выполняется следующая оценка:

$$
\begin{aligned}
|\langle G(t) \mid F(0)\rangle| \leqslant & 2\|F(0)\|_{L_{\xi, \beta}^{\infty}}\|G(0)\|_{C_{\gamma}} \sum_{s=0}^{\infty} \frac{\xi^{s}}{\gamma^{s}} \times \\
& \times \int_{\mathbb{R}^{s}} d p_{1} \ldots d p_{s} \exp \left\{-\beta \sum_{i=1}^{s} \frac{p_{i}^{2}}{2}\right\} \sum_{n=0}^{s} \frac{2^{n}}{n !} \times \\
& \times\left(C+\left(C_{1}+C_{2} t\right) s+t \sum_{i=1}^{s} p_{i}^{2}\right)^{n} C^{s-n} .
\end{aligned}
$$

Используя соотношение

$$
\begin{aligned}
(C+ & \left.\left(C_{1}+C_{2} t\right) s+t \sum_{i=1}^{s} p_{i}^{2}\right)^{n}= \\
& =\sum_{k=0}^{n} \frac{n ! C^{k}}{k !} \sum_{r=0}^{n-k} \frac{s^{r}}{r !}\left(C_{1}+C_{2} t\right)^{r} \frac{t^{n-k-r}}{(n-k-r) !}\left(\sum_{i=1}^{s} p_{i}^{2}\right)^{n-k-r}
\end{aligned}
$$


и неравенство

$$
\left(\sum_{i=1}^{s} p_{i}^{2}\right)^{n-k-r} \exp \left\{-\beta^{\prime} \sum_{i=1}^{s} \frac{p_{i}^{2}}{2}\right\} \leqslant(n-k-r) !\left(\frac{2}{\beta^{\prime}}\right)^{n-k-r}
$$

вычисляя интегралы по переменным импульса в выражении (3.10), получаем

$$
\begin{aligned}
|\langle G(t) \mid F(0)\rangle| \leqslant & 2\|F(0)\|_{L_{\xi, \beta}^{\infty}}\|G(0)\|_{C_{\gamma}} \sum_{s=0}^{\infty}\left(\frac{C \xi}{\gamma}\right)^{s}\left(\frac{2 \pi}{\beta^{\prime \prime}}\right)^{s / 2} \times \\
& \times \sum_{n=0}^{s}\left(\frac{2}{C}\right)^{n} \sum_{k=0}^{n} \frac{C^{k}}{k !} \sum_{r=0}^{n-k} \frac{s^{r}}{r !}\left(C_{1}+C_{2} t\right)^{r}\left(\frac{2 t}{\beta^{\prime}}\right)^{n-k-r},
\end{aligned}
$$

где $\beta=\beta^{\prime}+\beta^{\prime \prime}$. Положим $\widetilde{C}_{1}=\max \left(C_{1}, 1\right), \widetilde{C}_{2}=\max \left(C_{2}, 2 / \beta^{\prime}\right)$. Тогда для произвольного $t>0$ выполняются следующие неравенства:

$$
\widetilde{C}_{1}+\widetilde{C}_{1} t \geqslant 1, \quad\left(\widetilde{C}_{1}+\widetilde{C}_{2} t\right) \frac{\beta^{\prime}}{2 t} \geqslant 1,
$$

учитывая которые, имеем оценку

$$
\left(C_{1}+C_{2} t\right)^{r}\left(\frac{2 t}{\beta^{\prime}}\right)^{n-k-r} \leqslant\left(\widetilde{C}_{1}+\widetilde{C}_{2} t\right)^{n}
$$

Отсюда и из неравенств

$$
\sum_{k=0}^{n} \frac{C^{k}}{k !} \leqslant e^{C}, \quad \sum_{r=0}^{n-k} \frac{s^{r}}{r !} \leqslant e^{s}
$$

получаем, что оценка (3.12) принимает вид

$$
\begin{aligned}
|\langle G(t) \mid F(0)\rangle| \leqslant & 2 e^{C}\|F(0)\|_{L_{\xi, \beta}^{\infty}}\|G(0)\|_{C_{\gamma}} \sum_{s=0}^{\infty}\left(\frac{e C \xi}{\gamma}\right)^{s}\left(\frac{2 \pi}{\beta^{\prime \prime}}\right)^{s / 2} \times \\
& \times \sum_{n=0}^{s}\left(\frac{2}{C}\right)^{n}\left(\widetilde{C}_{1}+\widetilde{C}_{2} t\right)^{n}
\end{aligned}
$$

Меняя порядок суммирования в (3.15), окончательно получаем оценку (3.2).

Таким образом, из неравенства (3.2) следует, что при условиях (3.1) функционал (3.3) для среднего значения наблюдаемых корректно определен и соответствует функционалу (1.5) в случае регуляризованного представления (2.7) для решения задачи Коши двойственной иерархии ББГКИ. Утверждение доказано.

Заметим, что регуляризованное выражение (2.7) для начальных наблюдаемых аддитивного типа $G^{(1)}(0)=\left(0, a_{1}\left(0, x_{1}\right), 0, \ldots, 0, \ldots\right)$ имеет вид

$$
G^{(1)}(t)=\left(0, G_{1}^{(1)}\left(t, x_{1}\right), G_{2}^{(1)}\left(t, x_{1}, x_{2}\right), \ldots, G_{s}^{(1)}\left(t, x_{1}, \ldots, x_{s}\right), \ldots\right),
$$


где

$$
\begin{aligned}
G_{1}^{(1)}\left(t, x_{1}\right) & =\mathfrak{A}_{1}\left(t, x_{1}\right) a_{1}\left(0, x_{1}\right), \\
G_{s}^{(1)}\left(t, x_{1}, \ldots, x_{s}\right) & =\sum_{j=1}^{s} \sum_{\substack{Z \subset Y \backslash x_{j}, Z \neq \varnothing}}(-1)^{|Y \backslash Z|-1} \mathfrak{A}_{2}\left(t, x_{j},\{Z\}\right) a_{1}\left(0, x_{j}\right),
\end{aligned}
$$

$s=2,3, \ldots$.

Если $F(0) \in L_{\xi, \beta}^{\infty}, G^{(1)}(0) \in C_{\gamma, 0}$ и наблюдаемая $G_{s}^{(1)}\left(t, x_{1}, \ldots, x_{s}\right)$ определяется формулами (3.16), то функционал (1.5) принимает вид

$$
\begin{aligned}
& \left\langle G^{(1)}\right\rangle(t)=\left\langle G^{(1)}(t) \mid F(0)\right\rangle=\int_{\mathbb{R}^{1} \times \mathbb{R}^{1}} d x_{1} \mathfrak{A}_{1}\left(t, x_{1}\right) a_{1}\left(0, x_{1}\right) F_{s}\left(0, x_{1}, \ldots, x_{s}\right)+ \\
& +\sum_{s=2}^{\infty} \frac{1}{s !} \int_{\left(\mathbb{R}^{s} \backslash W_{s}\right) \times \mathbb{R}^{s}} d x_{1} \ldots d x_{s} \sum_{j=1}^{s} \sum_{\substack{Z \subset Y \backslash x_{j}, Z \neq \varnothing}}(-1)^{|Y \backslash Z|-1} \times \\
& \times \mathfrak{A}_{2}\left(t, x_{j},\{Z\}\right) a_{1}\left(0, x_{j}\right) F_{s}\left(0, x_{1}, \ldots, x_{s}\right)
\end{aligned}
$$

и выполняется следующая оценка:

$$
\begin{aligned}
\left|\left\langle G^{(1)}(0) \mid F(t)\right\rangle\right| \leqslant & \frac{2 e^{C+1} C \xi}{\gamma} \sqrt{\frac{2 \pi}{\beta^{\prime \prime}}}\|F(0)\|_{L_{\xi, \beta}^{\infty}}\left\|G^{(1)}(0)\right\|_{C_{\gamma}} \times \\
& \times \sum_{s=0}^{\infty}(2 e \xi)^{s}\left(\frac{2 \pi}{\beta^{\prime \prime}}\right)^{s / 2}\left(\widetilde{C}_{1}+\widetilde{C}_{2} t\right)^{s} .
\end{aligned}
$$

Данное неравенство подобно неравенству (3.15). Действительно, пусть $F(0) \in L_{\xi, \beta}^{\infty}$ и $G^{(1)}(0) \in C_{\gamma, 0}$. Для $(3.17)$ получаем

$$
\begin{gathered}
\int_{\mathbb{R} \times \mathbb{R}} d x_{1}\left|\mathfrak{A}_{1}\left(t, x_{1}\right) a_{1}\left(0, x_{1}\right)\right|\left|F_{1}\left(0, x_{1}\right)\right| \leqslant \frac{\xi C(t)}{\gamma}\|F(0)\|_{L_{\xi, \beta}^{\infty}}\left\|G^{(1)}(0)\right\|_{C_{\gamma}}, \\
C(t) \equiv\left|l_{1}(0)\right| \sqrt{\frac{2 \pi}{\beta}}+\frac{2 t}{\beta}
\end{gathered}
$$

и

$$
\begin{aligned}
& \sum_{s=2}^{\infty} \frac{1}{s !} \int_{\left(\Omega_{1}(0) \times \Omega_{s-1}(t)\right) \times \mathbb{R}^{s}} d x_{1} \ldots d x_{s} \times \\
& \times \sum_{j=1}^{s} \sum_{\substack{Z \subset\left\{x_{1}, \ldots, x_{s}\right\} \backslash x_{j}, Z \neq \varnothing}}\left|\mathfrak{A}_{2}\left(t, x_{j},\{Z\}\right) a_{1}\left(0, x_{j}\right)\right|\left|F_{s}\left(0, x_{1}, \ldots, x_{s}\right)\right| \leqslant \\
& \leqslant \frac{C \xi}{\gamma} e^{C+1} \sqrt{\frac{2 \pi}{\beta^{\prime \prime}}}\|F(0)\|_{L_{\xi, \beta}^{\infty}}\left\|G^{(1)}(0)\right\|_{C_{\gamma}} \sum_{s=1}^{\infty}(2 e \xi)^{s}\left(\frac{2 \pi}{\beta^{\prime \prime}}\right)^{s / 2}\left(\widetilde{C}_{1}+\widetilde{C}_{2} t\right)^{s},
\end{aligned}
$$

где использованы обозначения из оценки (3.2). Согласно (3.19) и (3.20) получаем оценку (3.18).

Заметим, что существование функционалов от $s$-кратных наблюдаемых рассматривалось для другого представления решения двойственной иерархии ББГКИ в работе [13].

5 Теоретическая и математическая физика, т. 162, № 3, 2010 г. 


\section{4. СРЕДНЕЕ ЗНАЧЕНИЕ НАБЛЮДАЕМЫХ: ЭВОЛЮЦИЯ СОСТОЯНИЙ}

Установим существование функционала (1.6) при условии, что эволюция состояний описывается регуляризованным представлением [11] для решения задачи Коши иерархии ББГКИ.

Пусть $\left\{x_{1}, \ldots, x_{s}\right\} \equiv Y \cup\left\{Y, x_{s+1}, \ldots, x_{s+n}\right\} \equiv X$, т.е.

$$
X \backslash Y=\left\{x_{s+1}, \ldots, x_{s+n}\right\}, \quad d x_{s+1} \ldots d x_{s+n} \equiv d(X \backslash Y) .
$$

Таким образом, $|X|=|Y|+|X \backslash Y|=s+n$.

Рассмотрим функционал (1.6) с состоянием $F_{s}\left(t, x_{1}, \ldots, x_{s}\right) \equiv F_{|Y|}(t, Y)$, которое определяется решением задачи Коши иерархии ББГКИ (регуляризованным решением) [11]:

$$
\begin{aligned}
& F_{|Y|}(t, Y)=\mathfrak{A}_{1}(-t,\{Y\}) F_{|Y|}(0, Y)+\sum_{n=1}^{\infty} \frac{1}{n !} \int_{\left(\mathbb{R}^{n} \backslash W_{n}\right) \times \mathbb{R}^{n}} d(X \backslash Y) \times \\
& \quad \times \sum_{\substack{Z \subset X \backslash Y, Z \neq \varnothing}}(-1)^{|X \backslash(Y \cup Z)|} \mathfrak{A}_{2}(-t,\{Y\},\{Z\}) F_{|X|}(0, X), \quad|X \backslash Y| \geqslant 1,
\end{aligned}
$$

где сумма под знаком интеграла берется по всем непустым подмножествам $Z$ множества $X \backslash Y$, оператор эволюции $\mathfrak{A}_{1}(-t,\{Y\})$ - это кумулянт первого порядка операторов эволюции $(2.1): \mathfrak{A}_{1}(-t,\{Y\})=S_{|Y|}(-t, Y)$, оператор эволюции $\mathfrak{A}_{2}(-t,\{Y\},\{Z\})-$ кумулянт второго порядка:

$$
\mathfrak{A}_{2}(-t,\{Y\},\{Z\})=S_{|Y \cup Z|}(-t, Y, Z)-S_{|Y|}(-t, Y) S_{|Z|}(-t, Z) .
$$

УтвеРЖДЕнИЕ 4.1. Для системы частии, с парным потенииалом взаимодействия $\Phi$, удовлетворяющим условиям (1.1), если $F(0) \in L_{\xi, \beta}^{\infty}, G(0) \in C_{\gamma, 0}$ и состояние определяется разложением (4.1), то при

$$
\begin{gathered}
\xi<\min \left(\frac{\gamma}{C}, \frac{2}{\left(2 \widetilde{C}_{1}+1\right)^{2}-1}\right) e^{-2 \beta b-1} \sqrt{\frac{\beta^{\prime \prime}}{2 \pi}} \\
0 \leqslant t<t_{0}, \quad t_{0} \equiv \frac{1}{2 \widetilde{C}_{2}}\left(-2 \widetilde{C}_{1}-1+\left(1+\frac{2 e^{-2 \beta b-1}}{\xi} \sqrt{\frac{\beta^{\prime \prime}}{2 \pi}}\right)^{1 / 2}\right),
\end{gathered}
$$

функционал (1.6) корректно определен и выполняется следующая оченка:

$$
\begin{aligned}
|\langle G(0) \mid F(t)\rangle| \leqslant & 2 e^{C}\|F(0)\|_{L_{\xi, \beta}^{\infty}}\|G(0)\|_{C_{\gamma}} \sum_{s=0}^{\infty}\left(\frac{C \xi e^{2 \beta b+1}}{\gamma} \sqrt{\frac{2 \pi}{\beta^{\prime \prime}}}\right)^{s} \times \\
& \times \sum_{n=0}^{\infty}\left(2 \xi e^{2 \beta b+1}\left(\widetilde{C}_{1}+\widetilde{C}_{2} t\right)\left(1+\widetilde{C}_{1}+\widetilde{C}_{2} t\right) \sqrt{\frac{2 \pi}{\beta^{\prime \prime}}}\right)^{n},
\end{aligned}
$$

где

$$
C=\max _{i=1, \ldots, s}\left|l_{i}(0)\right|
$$


u $l_{i}(0)$ - интервал из компакта $\Omega_{|Y|}(1.3)$, в котором лежит носитель функиии $G_{s}(0)$; остальные параметры $\widetilde{C}_{1}, \widetilde{C}_{2}, \beta$ и ь определяются так же, как в утверждении 3.1.

ДоказАтельство. Пусть $G(0) \in C_{\gamma, 0}$ и $F(0) \in L_{\xi, \beta}^{\infty}$. Предположим, что в начальный момент времени $t=0$ данные из множества $Y$ зафиксированы так, что наблюдаемая $G_{|Y|}(0, Y)$ локализуется в конфигурационном пространстве на компакте $\Omega_{|Y|}(0)=l_{1}(0) \times \cdots \times l_{|Y|}(0)(1.3)$.

Если маргинальные функции распределения $F_{s}\left(t, x_{1}, \ldots, x_{s}\right) \equiv F_{|Y|}(t, Y)$ в функционале (1.6) заданы разложением (4.1), то функционал (1.6) принимает вид

$$
\begin{aligned}
\langle G(0) \mid F(t)\rangle= & \sum_{s=0}^{\infty} \frac{1}{s !} \int_{\left(\mathbb{R}^{s} \backslash W_{s}\right) \times \mathbb{R}^{s}} d Y\left(\mathfrak{A}_{1}(-t,\{Y\}) F_{|Y|}(0, Y)+\right. \\
& +\sum_{n=1}^{\infty} \frac{1}{n !} \int_{\left(\mathbb{R}^{n} \backslash W_{n}\right) \times \mathbb{R}^{n}} d(X \backslash Y) \times \\
& \left.\times \sum_{\substack{Z \subset X \backslash Y, Z \neq \varnothing}}(-1)^{|X \backslash(Y \cup Z)|} \mathfrak{A}_{2}(-t,\{Y\},\{Z\}) F_{|X|}(0, X)\right) G_{|Y|}(0, Y) .
\end{aligned}
$$

В данном разложении область интегрирования по конфигурационным переменным $\mathbb{R}^{s} \backslash W_{s}$ ограничена, поскольку подынтегральное выражение конечно и отлично от нуля не во всем конфигурационном пространстве. Действительно, кумулянт первого порядка $\mathfrak{A}_{1}(-t,\{Y\})$ эквивалентен оператору $S_{|Y|}(-t, Y)(2.1)$, поэтому в (4.5) выражение $\mathfrak{A}_{1}(-t,\{Y\}) F_{|Y|}(0, Y) G_{|Y|}(0, Y)$ интегрируется не по всему конфигурационному пространству, а только по переменным из компакта $\Omega_{|Y|}(0), \Omega_{|Y|}(0) \subset \mathbb{R}^{s} \backslash W_{s}$, который при интегрировании сдвигается вдоль конфигурационной траектории и имеет конечный объем $V_{\Omega_{|Y|}(0)}=\prod_{i=1}^{|Y|}\left|l_{i}(0)\right|$.

Выражение

$$
\sum_{\substack{Z \subset X \backslash Y, Z \neq \varnothing}}(-1)^{|X \backslash(Y \cup Z)|} \mathfrak{A}_{2}(-t,\{Y\},\{Z\}) F_{|X|}(0, X)
$$

равно нулю вне области $\Omega_{|X \backslash Y|}(t)$ взаимодействия частиц с произвольными начальными условиями $X \backslash Y$ с частицами с фиксированными начальными условиями $Y$, $Y \in \Omega_{|Y|}(0)$, во временно́м интервале $[0, t)$. Это следует из того, что в таком случае выполняется свойство невзаимодействия $\mathfrak{A}_{2}(-t,\{Y\},\{Z\}) F_{|X|}(0, X)=0$ для всех $Z \subset X \backslash Y, Z \neq \varnothing$.

Таким образом, неравенство (4.5) приобретает вид

$$
\begin{aligned}
|\langle G(0) \mid F(t)\rangle| \leqslant & 2\|F(0)\|_{L_{\xi, \beta}^{\infty}\|G(0)\|_{C_{\gamma}} \times} \\
& \times \sum_{s=0}^{\infty} \frac{\left(\xi e^{2 \beta b}\right)^{s}}{\gamma^{s}} \int_{\Omega_{|Y|}(0) \times \mathbb{R}^{s}} d Y \exp \left\{-\beta \sum_{i=1}^{s} \frac{p_{i}^{2}}{2}\right\} \times \\
& \times \sum_{n=0}^{\infty} \frac{\left(2 \xi e^{2 \beta b}\right)^{n}}{n !} \int_{\Omega_{|X \backslash Y|}(t) \times \mathbb{R}^{n}} d(X \backslash Y) \exp \left\{-\beta \sum_{i=s+1}^{s+n} \frac{p_{i}^{2}}{2}\right\},
\end{aligned}
$$


где мы учли, что

$$
\begin{aligned}
& \left(\mathfrak{A}_{1}(-t,\{Y\})+\sum_{\substack{Z \subset X \backslash Y, Z \neq \varnothing}} \mathfrak{A}_{2}(-t,\{Y\},\{Z\})\right) \exp \left\{-\beta \sum_{i=1}^{s+n} \frac{p_{i}^{2}}{2}\right\} \leqslant \\
& \leqslant 2^{|X \backslash Y|} e^{2 \beta b|X|} \exp \left\{-\beta \sum_{i=1}^{s+n} \frac{p_{i}^{2}}{2}\right\} .
\end{aligned}
$$

Эта оценка следует из инвариантности гамильтониана системы $n$ частиц при действии оператора эволюции $S_{n}(-t)(2.1)$, из ограниченности области интегрирования по конфигурационным переменным (начальным конфигурационным компактом $\Omega_{|Y|}(0)$ и областью взаимодействия $\left.\Omega_{|X \backslash Y|}(t)\right)$, а также из условий (3.6) на потенциал взаимодействия $\Phi[1]$.

В правой части (4.6) область интегрирования по конфигурационным переменным $\Omega_{|Y|}(0) \times \Omega_{|X \backslash Y|}(t) \equiv \Omega_{|X|}\left(t, \Omega_{|Y|}(0)\right)$ имеет конечный объем $V_{\Omega_{|X|}(t)}$, для которого верна следующая оценка [11]:

$$
V_{\Omega_{|X|}(t)} \leqslant\left(C+\left(C_{1}+C_{2} t\right)(s+n)+t \sum_{i=1}^{s+n} p_{i}^{2}\right)^{n} C^{s},
$$

где

$$
C=\max _{i=1, \ldots, s}\left|l_{i}(0)\right|, \quad C_{1} \equiv 2 R, \quad C_{2} \equiv 2(4 b+1)
$$

и $b$ определяется в (3.6). Множитель перед $C^{s}$ в правой части оценки (4.7) можно представить в следующем виде:

$$
\begin{aligned}
(C+ & \left.\left(C_{1}+C_{2} t\right) s+t \sum_{i=1}^{s} p_{i}^{2}+\left(C_{1}+C_{2} t\right) n+t \sum_{i=s+1}^{s+n} p_{i}^{2}\right)^{n}= \\
= & \sum_{k=0}^{n} n ! \sum_{l=0}^{k} \frac{C^{l}}{l !} \sum_{m=0}^{k-l} \frac{s^{m}}{m !}\left(C_{1}+C_{2} t\right)^{m} \frac{t^{k-l-m}}{(k-l-m) !}\left(\sum_{i=1}^{s} p_{i}^{2}\right)^{k-l-m} \times \\
& \times \sum_{r=0}^{n-k} \frac{n^{r}}{r !}\left(C_{1}+C_{2} t\right)^{r} \frac{t^{n-k-r}}{(n-k-r) !}\left(\sum_{i=s+1}^{s+n} p_{i}^{2}\right)^{n-k-r} .
\end{aligned}
$$

После интегрирования по конфигурационным переменным в каждом члене ряда в правой части неравенства (4.6), учитывая (4.7), (4.8) и неравенство (3.11), вычисляем интегралы по импульсам. В результате получаем

$$
\begin{aligned}
|\langle G(0) \mid F(t)\rangle| \leqslant & 2\|F(0)\|_{L_{\xi, \beta}^{\infty}}\|G(0)\|_{C_{\gamma}} \sum_{s=0}^{\infty}\left(\frac{C \xi e^{2 \beta b}}{\gamma}\right)^{s}\left(\frac{2 \pi}{\beta^{\prime \prime}}\right)^{s / 2} \times \\
& \times \sum_{n=0}^{\infty}\left(2 \xi e^{2 \beta b}\right)^{n}\left(\frac{2 \pi}{\beta^{\prime \prime}}\right)^{n / 2} \sum_{k=0}^{n} \sum_{l=0}^{k} \frac{C^{l}}{l !} \sum_{m=0}^{k-l} \frac{s^{m}}{m !}\left(C_{1}+C_{2} t\right)^{m}\left(\frac{2 t}{\beta^{\prime}}\right)^{k-l-m} \times \\
& \times \sum_{r=0}^{n-k} \frac{n^{r}}{r !}\left(C_{1}+C_{2} t\right)^{r}\left(\frac{2 t}{\beta^{\prime}}\right)^{n-k-r}
\end{aligned}
$$

где $\beta=\beta^{\prime}+\beta^{\prime \prime}$. 
Введем обозначения $\widetilde{C}_{1}=\max \left(C_{1}, 1\right), \widetilde{C}_{2}=\max \left(C_{2}, 2 / \beta^{\prime}\right)$. Применяя неравенства $(3.13),(3.14)$ и

$$
\sum_{k=0}^{n}\left(\widetilde{C}_{1}+\widetilde{C}_{2} t\right)^{k} \leqslant\left(1+\widetilde{C}_{1}+\widetilde{C}_{2} t\right)^{n},
$$

приводим оценку (4.9) к виду (4.4). Из (4.4) следует, что если $\xi$ подчиняется условию (4.2), то разложение (4.5) сходится при $t \leqslant t<t_{0}$, где $t_{0}$ задано в формуле (4.3). Утверждение доказано.

Для наблюдаемых аддитивного типа $G^{(1)}(0)=\left(0, a_{1}\left(0, x_{1}\right), 0, \ldots, 0, \ldots\right)$ функционал (4.5) преобразуется в

$$
\left\langle G^{(1)}\right\rangle(t)=\left\langle G^{(1)}(0) \mid F(t)\right\rangle=\int_{\mathbb{R} \times \mathbb{R}} d x_{1} a_{1}\left(0, x_{1}\right) F_{1}\left(t, x_{1}\right)
$$

где $F_{1}\left(t, x_{1}\right)$ задается формулой $(4.1)$ для $|Y|=1$. Этот функционал корректно определен при

$$
\xi<\frac{2 e^{-2 \beta b-1}}{\left(2 \widetilde{C}_{1}+1\right)^{2}-1} \sqrt{\frac{\beta^{\prime \prime}}{2 \pi}}, \quad 0 \leqslant t<t_{0}
$$

\section{5. ЗАКЛЮЧЕНИЕ}

В настоящей работе доказано локальное по времени существование функционалов (1.5) и (1.6), описывающих системы бесконечного числа частиц, в случаях эволюции наблюдаемых и эволюции состояний при начальных условиях $F(0) \in L_{\xi, \beta}^{\infty}$ для некоторого класса наблюдаемых $G(0) \in C_{\gamma, 0}$, который определен в разделе 1.

Полученные результаты позволяют установить существование средних значений для более широких классов наблюдаемых $G(0) \in C_{\gamma, 0}$, чем рассматривались здесь (например, для локальных наблюдаемых [14], у которых средние значения описываются гидродинамическими уравнениями). Эти результаты можно также применить в случае так называемых интенсивных термодинамических наблюдаемых (например, числа частиц). Чтобы соответствующие функционалы для средних значений были корректно определены, необходимо вводить плотности этих наблюдаемых. Можно доказать существование средних значений интенсивных термодинамических наблюдаемых, используя полученные выше результаты в термодинамическом пределе [1].

Применяя метод продолжения решения иерархии ББГКИ, развитый в работах [1], [6], можно доказать глобальное по времени существование функционалов (1.5) и (1.6) для начальных условий, близких к равновесию. Более того, аналогично утверждениям 3.1 и 4.1 можно доказать существование функционалов для характеристик отклонения от средних значений наблюдаемых (например, дисперсии), описывающих флуктуации в неравновесных статистических системах.

Благодарности. Автор признательна профессору В. И. Герасименко за многочисленные научные дискуссии и ряд полезных комментариев. Эта работа частично поддержана Министерством образования и науки Украины (грант М/124-2007). 


\section{Список литературы}

[1] C. Cercignani, V.I. Gerasimenko, D. Ya. Petrina, Many-Particle Dynamics and Kinetic Equations, Math. Appl., 420, Kluwer, Dordrecht, 1997.

[2] C. Cercignani, R. Illner, M. Pulvirenti, The Mathematical Theory of Dilute Gases, Appl. Math. Sci., 106, Springer, New York, 1994.

[3] D. Ya. Petrina, Stochastic Dynamics and Boltzmann Hierarchy, Proc. Inst. Math. NASU. Math. Appl., 74, Inst. Math. NASU, Kyiv, 2008.

[4] H. Spohn, Large Scale Dynamics of Interacting Particles, Texts Monogr. Phys., Springer, Berlin, 1991.

[5] Н.Н. Боголюбов, Проблемы динамической теории в статистической физике, Гостехиздат, М.-Л., 1946.

[6] Д. Я. Петрина, ТМФ, 38:2 (1979), 230-250.

[7] Д. Я. Петрина, В. И. Герасименко, УМН, 38:5(233) (1983), 3-58.

[8] В. И. Герасименко, Д. Я. Петрина, ТМФ, 64:1 (1985), 130-149.

[9] Д. Я. Петрина, В. И. Герасименко, УМН, 45:3(273) (1990), 135-182.

[10] V. I. Gerasimenko, T. V. Ryabukha, M. O. Stashenko, J. Phys. A, 37:42 (2004), 9861-9872.

[11] T. V. Ryabukha, SIGMA, 2 (2006), 053.

[12] В. I. Герасименко, Т. В. Рябуха, Допов. НАН Укр., 3 (2003), 16-22.

[13] G. Borgioli, V. Gerasimenko, Riv. Mat. Univ. Parma (7), 4 (2001), 251-267.

[14] М. М. Боголюбов, "Рівняння гідродинаміки в статистичній механіці", АН УРСР. Збірник прачь Інституту математики, 10 (1948), 41-59.

Поступила в редакцию 7.04.2009 